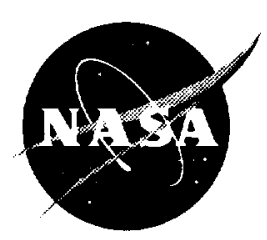

\title{
The Physics of Hard Spheres Experiment on MSL-1: Required Measurements and Instrument Performance
}

Michael P. Doherty

Lewis Research Center, Cleveland, Ohio

Christian T. Lant

ADF Corporation, Brook Park, Ohio

Jerri S. Ling

Lewis Research Center, Cleveland, Ohio

Prepared for the 36th Aerospace Sciences Meeting sponsored by the American Institute of Aeronautics and Astronautics Reno, Nevada, January 12-15, 1998

National Aeronautics and Space Administration

Lewis Research Center 


\section{Acknowledgments}

The authors wish to acknowledge Prof. Paul M. Chaikin, Prof. William B. Russel, Dr. Jixiang Zhu, Zhengdong Cheng, and See-Eng Phan of the Principal Investigator's team, Prof. David S. Cannell,

Dr. Anthony E. Smart, William L. Shiley, Eric E. Anderson, Michael T. Lehto, John P. Bowen, and

J. Michael Shoemaker of the NASA LeRC hardware development team, and also to the several hundred people in all at LeRC and its contractors and subcontractors who contributed to the design, fabrication, assembly, testing, integration, operation, and public relations activities critical to the success of this space flight experiment.

Trade names or manufacturers' names are used in this report for identification only. This usage does not constitute an official endorsement, either expressed or implied, by the National Aeronautics and Space Administration.

Available from

NASA Center for Aerospace Information 800 Elkridge Landing Road

Linthicum Heights, MD 21090-2934

Price Code: A03
National Technical Information Service 5287 Port Royal Road Springfield, VA 22100

Price Code: A03 


\title{
THE PHYSICS OF HARD SPHERES EXPERIMENT ON MSL-1: REQUIRED \\ MEASUREMENTS AND INSTRUMENT PERFORMANCE
}

\author{
Michael P. Doherty*, Christian T. Lantt, and Jerri S. Ling* \\ *NASA Lewis Research Center \\ 21000 Brookpark Rd., Cleveland, $\mathrm{OH} 44135$ \\ †ADF Corporation \\ 3003 Aerospace Parkway, Brook Park, OH 44142
}

\begin{abstract}
The Physics of HArd Spheres Experiment (PHaSE), one of NASA Lewis Research Center's first major light scattering experiments for microgravity research on complex fluids, flew on board the Space Shuttle's Microgravity Science Laboratory (MSL-1) in 1997. Using colloidal systems of various concentrations of micronsized plastic spheres in a refractive indexmatching fluid as test samples, illuminated by laser light during and after crystallization, investigations were conducted to measure the nucleation and growth rate of colloidal crystals as well as the structure, rheology, and dynamics of the equilibrium crystal. Together, these measurements support an enhanced understanding of the nature of the liquid-to-solid transition. Achievement of the science objectives required an accurate experimental determination of eight fundamental properties for the hard sphere colloidal samples. The instrument design met almost all of the original measurement requirements, but with compromise on the number of samples on which data were taken. The instrument performs 2-D Bragg and low angle scattering from $0.4^{\circ}$ to $60^{\circ}$, dynamic and single-channel static scattering from $10^{\circ}$ to $170^{\circ}$, rheology using fiber optics, and white light imaging of the sample.
\end{abstract}

As a result, $\mathrm{PHaSE}$ provided a timely microgravity demonstration of critical light scattering measurement techniques and hardware concepts, while generating data already showing promise of interesting new scientific findings in the field of condensed matter physics.

M. P. Doherty, member of AIAA, and J. S. Ling, member of AIAA, are with the NASA Lewis Research Center, Cleveland, $\mathrm{OH}$ and $\mathrm{C}$. T. Lant is with ADF Corporation, Brookpark, $\mathrm{OH}$.

\section{INTRODUCTION}

The Physics of HArd Spheres Experiment (PHaSE) flew on board the Space Shuttle's Microgravity Science Laboratory (MSL-1) twice in 1997 (STS-83 and STS-94). PHaSE is a fluid physics experiment whose purpose is to investigate the behavior and physical properties of a hard sphere system in microgravity. The fundamental physics of the molecular phenomenon of the liquid-to-solid phase transition (essentially, the dynamics of solidification) can be studied using a colloidal system of micron-sized hard plastic spheres which have been tailored to reduce all attractive forces. A colloidal system, or colloid, consists of fine particles, often having complex interactions, suspended in a liquid. Paint, ink, and milk are examples of colloids found in everyday life. At specific concentrations (i.e., volume fractions), uniformly sized (i.e., monodisperse) hard sphere colloids demonstrate the behavior of crystal nucleation and growth, driven purely by configurational entropy due to constraints on the packing of the impenetrable particles at high densities. The PHaSE experiment performs light scattering and rheological measurements on the colloidal system, with the goal being to probe the essential features of the hard sphere disorder-order transition and the properties of the resulting phase.

Atomic interactions are very complex, with much more still to be learned. Three of the traditional experimental difficulties are that the interesting thermodynamic behavior is complicated by electromagnetic interactions, the phase transition occurs on an extremely fast timescale, and the length scale of the atomic arrangement 
requires $x$-ray wavelengths to resolve. One powerful approach to avoid these difficulties is to study a system of simpler, larger particles that behaves in a manner similar to the atomic system. PHaSE uses colloidal systems of microscopic plastic spheres that are capable of being probed using visible light. Even monodisperse spheres that cannot penetrate each other and do not otherwise interact (hard spheres) share a fundamental characteristic with atomic systems - both undergo a transition from a disordered liquid state to an ordered solid state under the proper conditions, just as when water molecules become ordered to form ice. The literature is replete with studies of the thermodynamics of hard sphere systems. ${ }^{1,2,3}$ Recent publications have stated the following: "The hard-sphere system is probably the simplest model that reproduces qualitatively the characteristics of the melting transition of real systems," and "Colloidal crystals provide scientists with a way to test how atoms interact and what they do during phase transitions."

When monodisperse hard spheres suspended in a fluid reach a certain volume fraction (i.e., the fraction of the total sample volume actually filled by the spheres), the particle-fluid mixture changes from a disordered fluid state, in which the spheres are randomly moving, to an ordered crystalline state in which they are arranged periodically. The thermal energy of the spheres causes them to jostle each other until they form ordered arrays, or crystals, because this arrangement allows each sphere the most freedom of movement. If the hard sphere volume fraction is too high, on Earth the rearrangement seems not to occur. Such hard sphere systems behave like glass. Figure 1 shows how the hard spheres arrange themselves in the different phases, and the volume fractions at which these phases occur on Earth.

The best way to quantify the behavior of hard sphere systems is to scatter laser light off of them. To produce good quality scattered light, the fluid and the spheres must have nearly the same index of refraction and the diameter of the spheres must be comparable to the wavelength of the laser light. Unfortunately, spheres having the same density and refractive index as the fluid cannot be synthesized at this time, which,

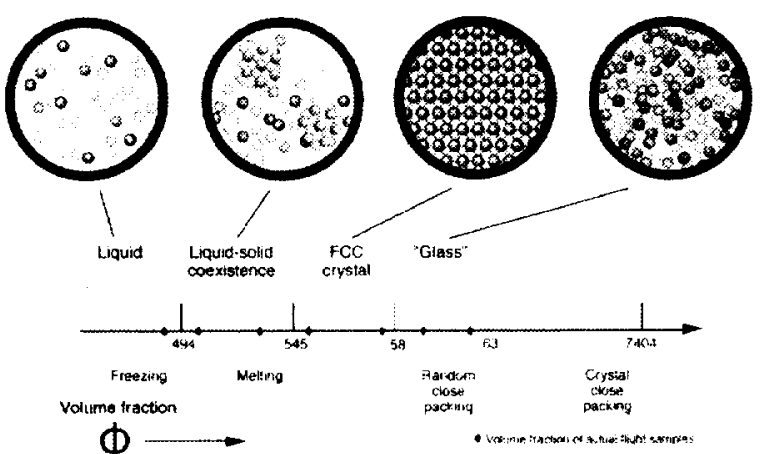

Fig. 1 Equilibrium phase diagram

on Earth, causes the spheres more dense than the fluid to sink and the spheres less dense than the fluid to float. Because, on Earth, the density mismatch in colloidal systems causes significant settling over the crystallization period, they are a poor model of the equilibrium (long term) atomic system, where the effect of gravity is truly negligible.

The solution to this gravitational problem is, of course, to conduct the experiment in space. PHaSE provided a compact experimental platform to perform a variety of laboratory-quality light scattering measurements remotely in a microgravity environment. Data was gathered using several light scattering techniques, and comparison with measurements performed under normal gravity conditions will distinguish the true equilibrium behavior from the effects due to gravity.

Eight samples flew on PHaSE. Seven of the samples ranged in volume fraction from 0.483 to 0.624 to cover the range of interest, while one sample, having a volume fraction of 0.019 , was utilized for instrument calibration. Figure 1 indicates with diamond markers the volume fraction of each of the seven flight samples. These particular flight samples were chosen to span the range from the disordered fluid, across the freezing and melting transitions, and into the glass region.

The Colloidal Disorder-Order Transition (CDOT) experiment, a precursor to PHaSE, yielded dramatic results. ${ }^{6,7,8}$ Large, dendritic crystallites grew in samples which, on Earth, produce a sediment of much smaller crystallites. In addition, a sample which was glassy on Earth 
actually crystallized in microgravity, contrary to prior Earth-based observations. Gravity thus appears to play a significant and unexpected role in the formation and structure of colloidal crystals. Through its more quantitative data, PHaSE will provide the Principal Investigators a deeper understanding of the nature of phase transitions. The knowledge derived will add to the understanding of condensed matter, which helps scientists reduce the trial and error involved in developing new and better materials. Industries dealing with semiconductors, electrooptics, ceramics, and composites are just a few that may benefit from this knowledge.

PHaSE was conceived by Principal Investigators (Pis), Professors Paul M. Chaikin and William B. Russel with Research Scientist Dr. Jixiang Zhu, all from Princeton University. It was designed, built, and tested by NYMA Corporation, ADF Corporation, and the Spectron Division of the Titan Corporation, with significant contributions from Wyle Laboratories, Inc. and Hughes Danbury Optical Systems, Inc., under the direction of Project Manager Michael P. Doherty, Project Scientist Jerri S. Ling, and Facility Scientist William V. Meyer of the Microgravity Science Division at the NASA Lewis Research Center (LeRC) in Cleveland, Ohio. This experiment is supported by Office of Life and Microgravity Sciences and Applications at NASA Headquarters.

This paper identifies the PIs' required measurements and their accuracies, explains the design features of the flight instrument to enable the measurements to be made to their required accuracies, and reports on how well the PHaSE flight instrument performed in flight.

\section{REQUIRED MEASUREMENTS AND THEIR ACCURACIES}

The science objectives of the Physics of HArd Spheres Experiment include: (1) quantifying the kinetics of the nucleation and growth for each phase, (2) determining the equilibrium structure associated with each phase, (3) quantifying the dynamics of Brownian fluctuations for each phase, and (4) quantifying the static elastic modulus and dynamic viscosity of the crystalline phase. The science objectives require an accurate experimental determination of eight fundamental properties for the hard sphere colloidal samples. These properties are the nucleation and growth rates (to $50 \%$ accuracy), the lattice constant (to $0.3 \%$ accuracy) and crystal arrangement, the diffusion factor (to $10 \%$ accuracy) and root mean square displacement (to $2 \%$ accuracy), and the elastic constant and dynamic viscosity (to $10 \%$ accuracy).

The nucleation rate is the rate of change with time in the number of individual crystallites, while the growth rate is the rate of change with time of crystallite size. The lattice constant is the centerto-center spacing between the ordered hard spheres, which is dependent, in part, upon the crystal arrangement (e.g. Face-Centered Cubic, Random Hexagonal Close Packed). The diffusion factor (the ability of a single particle to move around within its lattice cage, or to diffuse completely out of its lattice cage) and the root mean square displacement (measure of the Gaussian distribution of the distance a particle moves from its lattice site) are two separate properties associated with the equilibrium structure and are an important means to quantify the dynamics of Brownian fluctuations. The elastic constant and the dynamic viscosity are measures, respectively, of the stiffness and damping characteristics of the resulting crystal.

\section{DESIGN FEATURES OF THE INSTRUMENT}

The above measurements defined the instrument requirements including full-field large-ensemble Bragg and low angle measurements, multi-angle dynamic and static light scattering, measurements of shear modulus, and color imaging. Integrating these capabilities to work well on the same specimen, while operating remotely in a confined space, required a novel design of optics, detectors, and actuators.

PHaSE was developed on a compressed time schedule. The impetus for the compressed time 
schedule was: 1) the maturity of Light

Scattering as a technology was recognized, ${ }^{9,10}$ implying that the tools and techniques wouldn't require further development; 2) NASA was attempting to reduce the cost and time to acquire data for its science missions; and 3) MSL-1 was the last flight opportunity for such an experiment prior to the International Space Station (ISS). Flying PHaSE in 1997 on a Shuttle Spacelab mission was seen to provide a rigorous test of the benefits of microgravity complex fluids research as well as an early opportunity to verify critical Light Scattering Instrument (LSI) hardware concepts for use on ISS. The hardware development team was successful in developing the flight hardware for this first major light scattering experiment in a 32month timeframe (from Science Concept Review to flight hardware readiness). For comparison, the precedence for similar flight hardware development had been 45-54 months. The implementation of the project on a compressed timescale included an abridged hardware model development sequence, classification of the experiment as a Class D (low cost, higher risk) payload, utilization of as much existing design and hardware heritage as possible, and extremely good communication and tight coordination within the hardware team, the project team, and the Microgravity Science Division.

The PHaSE flight system consists of three modules: Test Section, Avionics Section, and Power Drawer (Figure 2). ${ }^{11}$

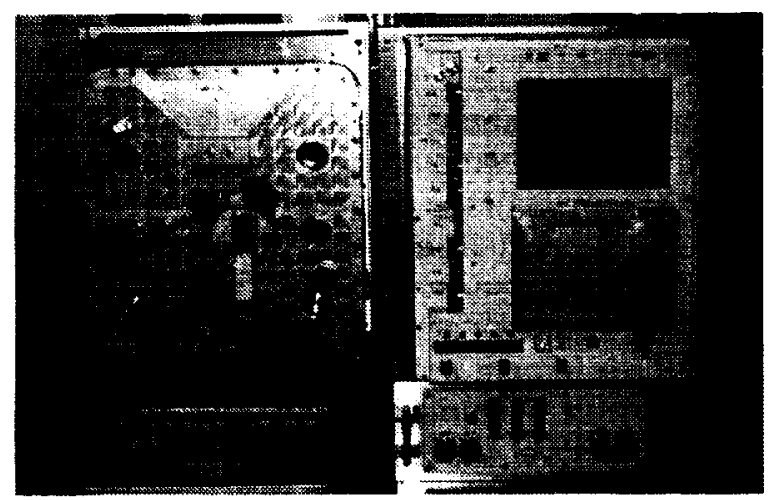

Fig. 2. PHaSE flight hardware. Left to right: Test Section, Avionics Section (above), and Power Drawer (below)

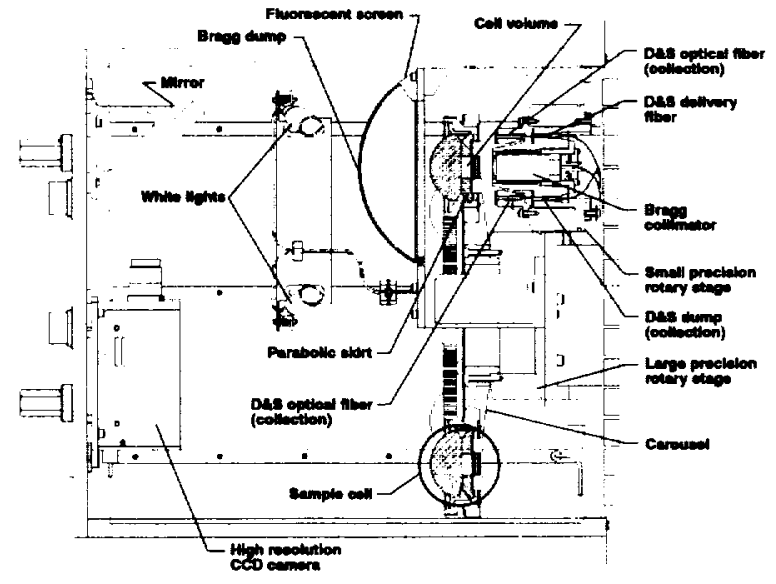

Fig. 3. Test section

The Avionics Section and Power Drawer provide support electronics to control and monitor the system, while the Test Section is where the key measurements are made. The following paragraphs will, therefore, concentrate on the Test Section design and operation.

The Test Section of the instrument payload is housed in a Universal Small Experiment Container, supplied by Wyle Laboratories, Inc. ${ }^{12}$ The suite of measurements performed in the Test Section include: 1) 2-D Bragg and low angle scattering from $\theta=0.4^{\circ}$ to $60^{\circ}$ at up to $0.1^{\circ}$ resolution, where $\theta$ is the scattering angle; 2) dynamic and single-channel static measurements from $\theta=10^{\circ}$ to $170^{\circ}$ at $0.01^{\circ}$ resolution; 3) rheology using fiber optics; and 4) white light imaging of the sample. These scattering angle ranges correspond to $0.9 \leq q d \leq 12.2$ for $2-D$ Bragg and low angle scattering and $2.1 \leq q d \leq 24.3$ for dynamic and static scattering. Here $\mathrm{q} \equiv\left[4 \pi \mathrm{n} / \lambda_{o}\right] \times \sin (\theta / 2)$ is the scattering wavevector, $\mathrm{n}$ is the index of refraction of the medium, $\lambda_{o}$ is the wavelength of the incident light, and $d=684 \mathrm{~nm}$ is the particle diameter.

Figure 3, a schematic drawing of the Test Section, shows an edge view including the 
carousel containing eight sample cells, with two cells visible in section. The cell uppermost in the figure is in the test location. The glass cells, index matched to the samples, contain 3.2 milliliter samples of cis-decalin, tetralin, and polymethyl-methacrylate (PMMA) spheres, with the spheres having a thin coating of polyhydroxystearic acid (PHSA) to minimize attractive forces. The samples are approximately $25 \%$ decalin, $25 \%$ tetralin, and $50 \%$ PMMA spheres, by volume. The exact ratio of the components for each sample is adjusted to achieve the required volume fraction of solid particles to total volume.

Except for the white light imaging, all measurements are made at a single location into which each sample may be moved by a rotaryactuated stage. In this position, the sample is mechanically oscillated to homogenize (or shear melt) it before beginning an experiment sequence. Laser light of $532 \mathrm{~nm}$ wavelength is then introduced into the specimen cell volume ( $20 \mathrm{~mm}$ diameter by $10 \mathrm{~mm}$ deep) in one of two ways. The Bragg scattering optics produces an $8 \mathrm{~mm}$ ( $e^{-2}$ intensity diameter) collimated laser beam with a Gaussian profile. This $4 \mathrm{~mW}$ beam passes axially through the sample cell to illuminate a large volume of the specimen to average over many crystallites. The dynamic and static scattering optics produces a second, tightly focused laser beam that is totally internally reflected from the cell skirt to enter the specimen radially. In each case, the laser source in the Avionics Section is brought to the Test Section via a single mode, polarization maintaining fiber optic cable.

Light scattered into a Bragg pattern by the crystallizing PMMA spheres is focused by the hemispherical exit surface of the cell onto a concentric fluorescent screen, while the unscattered light is focused by the same optics into a beam dump in the center of the screen. Mounted on the far wall of the container is a $1300 \times 1000$ pixel Charge Coupled Device (CCD) camera which, via a flat steering mirror, records an image of the spherical screen upon which the Bragg scattered light is incident. The concentric spherical design maps onto the surface of the screen all light scattered by the sample from $\theta=0.4^{\circ}$ (determined by the outer limit of the beam dump) to $\theta=60^{\circ}$ (the outer limit of the screen).

Nucleation and growth rates of all samples are both measured using 2-D Bragg and low angle scattering. High resolution CCD images taken at regular intervals beginning shortly after shear melting provide quantitative measurement of the nucleation and growth of the crystals. These digital images of the rings of scattered light on the screen subsequently are azimuthally averaged and plotted as intensity vs. scattering angle. The number and volume of the crystallites is derived by measuring the maximum signal intensity at low angles and by integrating the signal intensity under the first Bragg peak. The nucleation and growth rates follow simply from the rate of change of these measurements. The measurement of nucleation and growth rates to $50 \%$ accuracy requires that the 2-D Bragg and low angle through-system angular resolution, $\Delta \theta$, be $\leq 0.25^{\circ}$ and the minimum observable angle, $\theta_{\min }$, be equal to $0.25^{\circ}$.

The lattice constant and crystal arrangement are also determined primarily by 2-D Bragg scattering, but also by the single-channel static measurements. These Static Light Scattering (SLS) measurements from $\theta=10^{\circ}$ to $170^{\circ}$ provide measurement of Bragg scattering maxima beyond $\theta=60^{\circ}$ as well as independent corroboration of the Bragg scattering from $\theta=10^{\circ}$ to $60^{\circ}$. The lattice constant is derived from the location of the first Bragg ring, and the crystal arrangement is determined by the number, location, and spacing of the first several Bragg maxima, found by Bragg scattering and SLS. The measurement of the crystal lattice constant to a $0.3 \%$ accuracy requires that the $2-D$ Bragg through-system angular resolution, $\Delta \theta$, be $\leq$ $0.2^{\circ}$. 


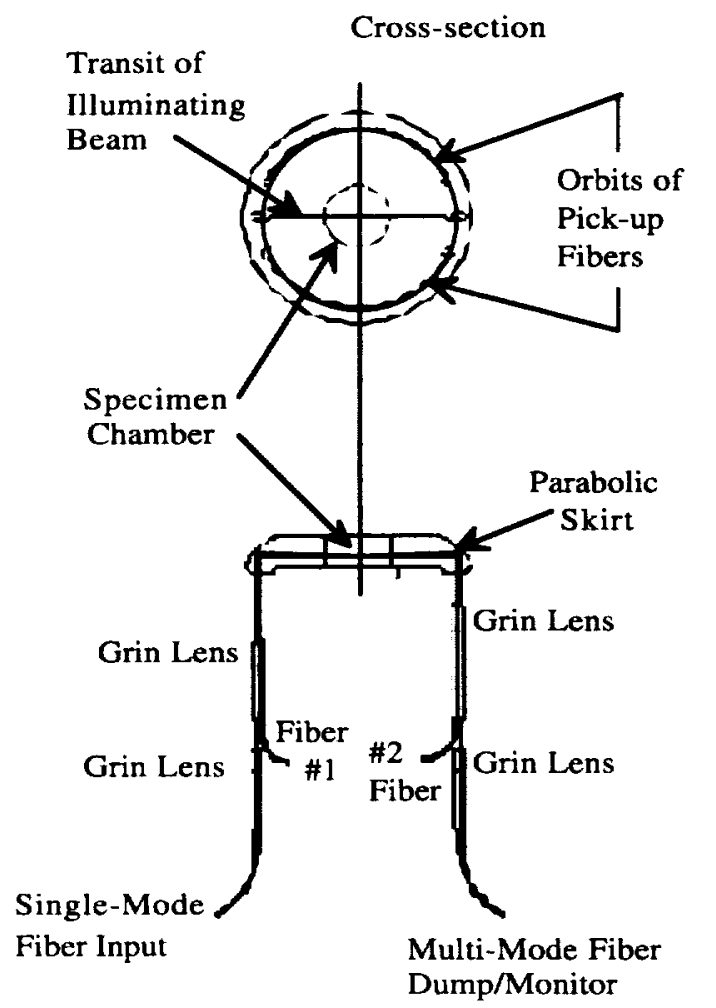

Fig.4. Dynamic and static scattering optics

Figure 4 shows how the $3 \mathrm{~mW}$ laser beam is introduced for dynamic and static (D\&S) measurements (normally performed after kinetically-driven growth is complete) via a singlemode optical fiber terminated by a quarter-pitch graded index (GrIn) lens. Total internal reflection from the parabolic-shaped cell skirt focuses the D\&S beam to a $100 \mu \mathrm{m}$ ( $e^{-2}$ intensity diameter) Gaussian waist in the center of the cell. The directly transmitted beam is collected by a Grin lens into the $60 \mu \mathrm{m}$ diameter core of a multimode fiber. The beam is thus absorbed to suppress stray light and is led to an avalanche photodiode (APD) detector for laser power monitoring. Scattered light for dynamic and static measurements is collected by two singlemode optical fibers terminated by Grin lenses. A precision stage scans the collection optics over scattering angles from $\theta=10^{\circ}$ to $170^{\circ}$. For each fiber receiver, the scattered light is coupled through a narrow-band filter into a multi-mode fiber and thence to the Avionics Section and its APD. The APDs are the single photon counting
SPCM-AQ models manufactured by EG\&G Optoelectronics, Canada ${ }_{1}^{12}$ which have a very high quantum efficiency, active quenching, and dark counts around 50 counts per second. Dynamic correlations and static count rates are then measured and reported by two correlators manufactured by Brookhaven Instruments Corporation. ${ }^{12}$ The parabolic optics allow examination of a broad range of scattering angles in the confined space permitted. The focus of the parabola is at the intersection between the illumination beam and the pickup fiber focus. Single mode pickup fibers terminated by quarter-pitch GrIn lenses restrict the k-vector of the collected light, ${ }^{13}$ but in a smaller space than a more conventional pair of apertures. In this system the azimuthal range is limited only by mechanical interference between the rotating and stationary fiber housings. The highly polished antireflection-coated cells exhibit negligible flare above the mechanical limit of $10^{\circ}$ scattering angle.

The diffusion factor and the root mean square displacement are determined from autocorrelation functions generated from highly ensemble-averaged Dynamic Light Scattering (DLS) measurements, taken at numerous scattering angles. References for DLS, also called photon correlation spectroscopy, are listed. ${ }^{14,15,16,17}$ The diffusion factor is related to the exponential decay of the autocorrelation function, ${ }^{18}$ while the root mean square displacement is related to the value to which the autocorrelation function decays at large delay times. At small scattering angles the temporal fluctuations decay at a rate governed by the gradient diffusion coefficient, while at larger scattering angles, the self diffusion coefficient controls the rate of decay. ${ }^{19}$ The measurement of the diffusion factor and the root mean square displacement to a $10 \%$ accuracy and $2 \%$ accuracy, respectively, has implications on the ensemble averaging via rotation of the sample. The desired accuracy requires that the product of the scattering wavevector and size of the scattering volume for dynamic and static scattering enables that at least 2500 ensembles are generated to sufficiently average the statistics. 


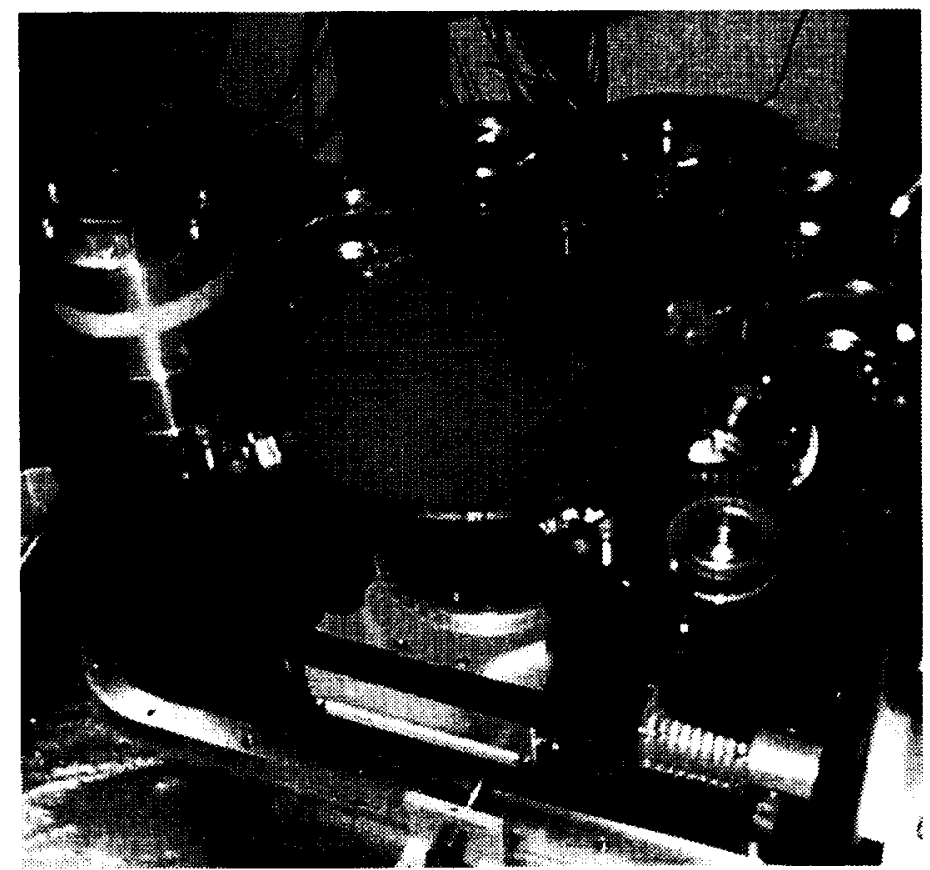

Fig. 5. View of test section with fluorescent screen

Adjacent to the primary test location is a color CCD camera, which records and downlinks opalescent images of the samples at a resolution better than $50 \mu \mathrm{m} /$ picture element (pixel) using a 768 by 494 pixel array. These images are used to evaluate the crystallization of the samples at various times during the mission.

Figure 5 shows an interior view of the Test Section without the enclosure walls.

Prominently visible are the Bragg screen and Bragg beam dump (center), the carousel of cells, the fiber receiver precision rotary stage (bottom center), and mix/melt DC servo motor (upper left). The DC servo motor performs the four functions of slowly rotating the cell for ensemble averaging for the static measurements, stepping the cell for ensemble averaging for the dynamic measurements, gently oscillating the cell for rheology measurements, and vigorously oscillating the samples to mix (or melt) them before beginning an experiment sequence.

Rheology is another investigation conducted once kinetically-driven growth is complete. Rheology measurements are made by performing DLS via the parabolic skirt while slowly oscillating the sample cell by a known frequency (at low amplitude), to enable measurements to be taken of the mechanical frequency response of the crystal. The elastic constant is derived from the value of the fundamental frequency of the crystal while the dynamic viscosity is derived from the width of the frequency response curve at half the amplitude of the fundamental frequency. The measurement of the elastic constant and the dynamic viscosity to a $10 \%$ accuracy requires a resolution of $0.1 \mathrm{Hertz}$ in the drive frequency.

\section{INSTRUMENT PERFORMANCE IN FLIGHT}

The MSL-1 mission flew on April 4-8, 1997 and again July $1-17,1997$. The instrument conducted 85 runs and collected high resolution images and correlation data almost continuously for a 336 hour (nearly two week) duration. Since PHaSE had been designed to be remotely operated from the ground, astronaut resources were only required for a total of five occasions adding up to less than $\mathbf{3 0}$ minutes of crew time. This included time to activate and deactivate PHaSE, to perform several instrument system reboots, and once to program a parameter 


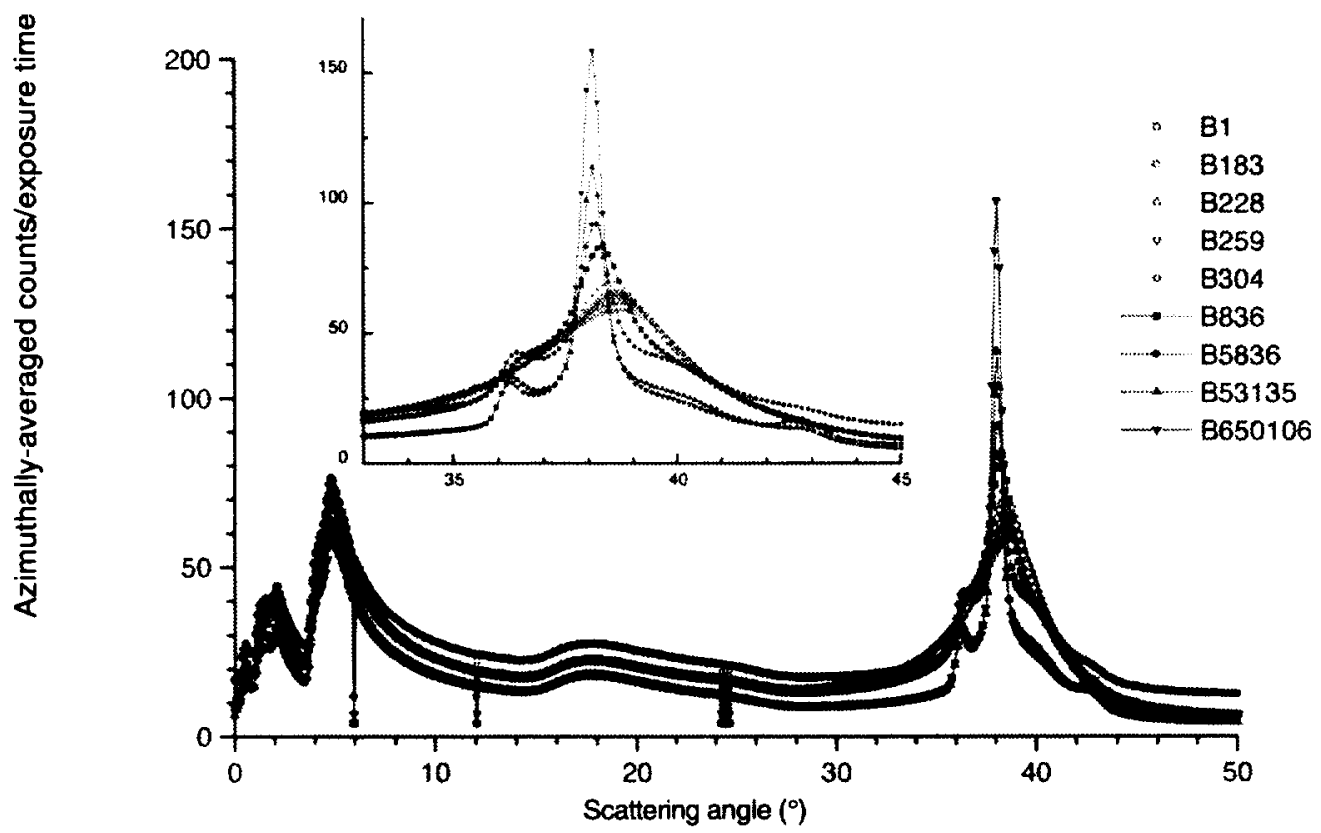

Fig. 6. Azimuthally averaged Bragg images (from $\theta=0^{\circ}$ to $50^{\circ}$ ) taken of Sample 4 over a period from 1 second to 650,106 seconds (or $\approx 7.5$ days) after shear melting

change to the flight hardware during an extended, but temporary, loss-ofcommunication period.

The final instrument design met almost all of the original measurement requirements, but with compromise on the number of samples on which data were taken. Alternative procedures and designs were found in all but three areas where instrument performance fell (or appeared to fall) short of the specified requirements (unfortunately, modifications to address these remaining problems could not be implemented due to the compressed development schedule): 1) low angle measurements were compromised by excessive signal on the screen at low angles and an "as-built" minimum observable scattering angle that fell slightly short of the requirement, 2) a drop in delivered laser power in the D\&S beam between the time of hardware turnover and flight reduced the quality of some DLS and SLS measurements, and 3) four out of eight samples developed bubbles following the final thermal tests of the assembled system. This loss of four samples precluded characterization of the liquid and glassy regions of the phase diagram. (Ironically, testing fewer samples allowed time for much deeper characterizations than originally intended of those that remained intact - samples 3 (coexistence region), 4 (crystalline region), and 5 (crystalline region)). Each of these problems is being currently addressed under the design of a second generation light scattering instrument.

Color images were used to confirm sample status during flight. These images supported the more quantitative high resolution Bragg images in monitoring and measuring the onset and progress of sample crystallization.

High resolution CCD Bragg images taken on orbit at regular intervals beginning shortly after shear melting of each sample provide quantitative measurement of the nucleation and growth of the crystals. Because the throughsystem angular resolution was validated to be $\Delta \theta= \pm 0.16^{\circ}$ at a scattering angle of $\theta=8.7^{\circ}$, $\Delta \theta= \pm 0.17^{\circ}$ at a scattering angle of $\theta=32^{\circ}$, and 


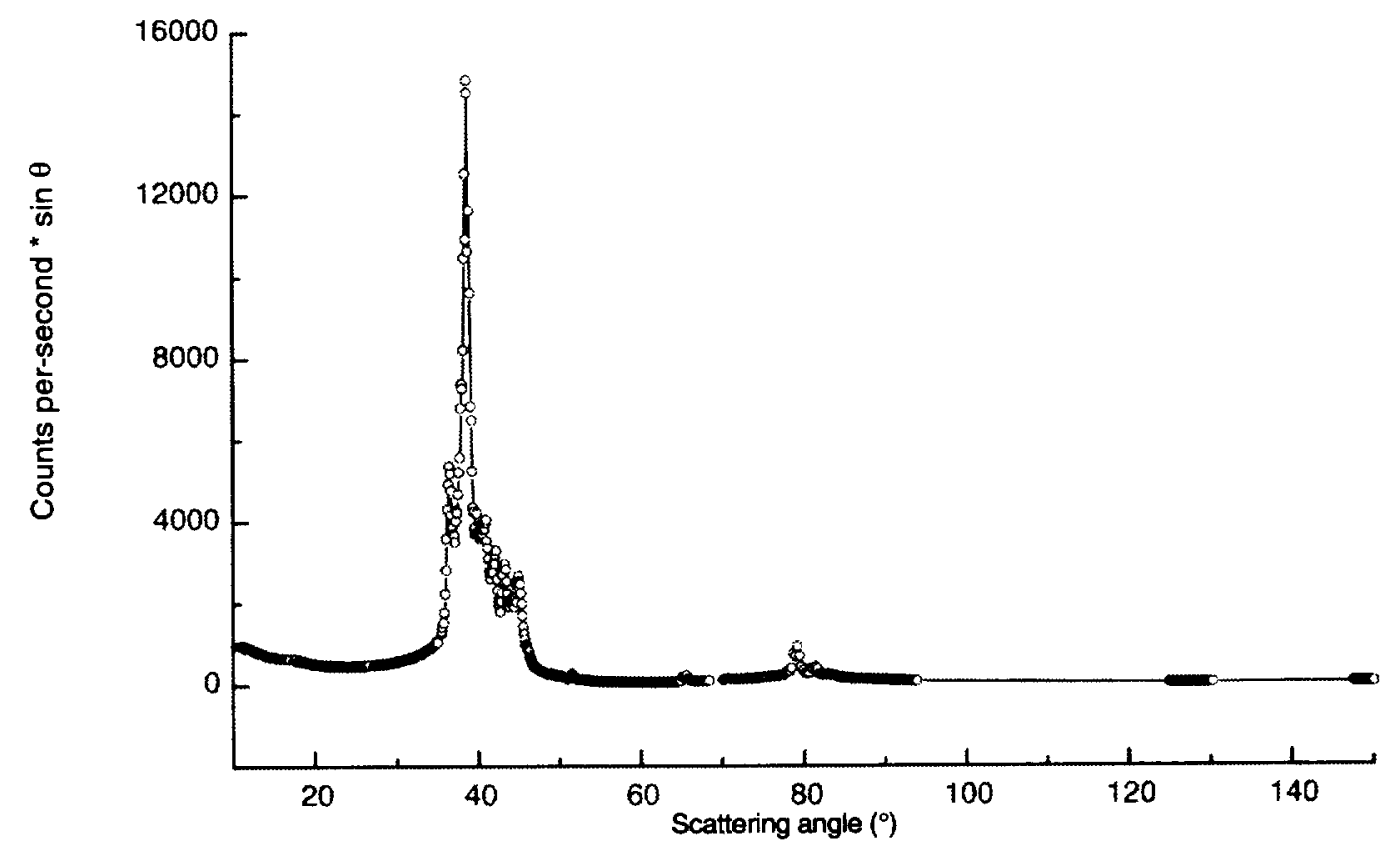

Fig. 7. Static Light Scattering on Sample 4 from $10^{\circ}$ to $150^{\circ}$

$\Delta \theta= \pm 0.35^{\circ}$ at a scattering angle of $\theta=54.8^{\circ}$ the first criterion for achieving the required accuracies for nucleation and growth rates, $\Delta \theta \leq$ $0.25^{\circ}$ for Bragg scattering, was met. Figure 6 is an overlay of nine azimuthally averaged Bragg images (from $\theta=0^{\circ}$ to $50^{\circ}$ ) taken of Sample 4 over a period from 1 second to 650,106 seconds (or $\approx 7.5$ days) after shear melting, clearly showing the presence of, as a well as the subsequent increase and eventual sharp definition of, the first Bragg peak for this sample. (The inset on the figure provides more detailed definition of the growth in the region of the Bragg peak.) The height of the peak and its width yield valuable information about the size and number of crystallites in the sample at each time. By at least 53,135 seconds (or $\approx 14.5$ hours) after shear melting (the curve denoted by B53135), the peak's width has considerably narrowed and grown higher, a phenomenon corresponding to the size of the crystallites increasing. By analyzing how these scattering curves change during time intervals of interest, the PIs will be able to derive growth rates for the crystal.

Due partly to the excessive signal at low angles (behavioral shortcoming) and partly to the design not quite achieving the minimum observable scattering angle requirement $\left(\theta_{\min }=0.25^{\circ}\right)$, the instrument was not able to make unambiguous measurements on the samples to the required minimum angle. (In fact, three neutral density filters were required on the screen from $\theta=0.3^{\circ}$ to $4^{\circ}$ to compress the dynamic range of the signal to accommodate camera exposures lengthy enough to measure Bragg scattering.) The inadequacy in making the low angle measurement in this experiment may preclude the determination of scaling factors from which the PIs can quantify both nucleation and growth rates in an absolute sense. The behavioral shortcoming in instrument performance initially appeared attributable to flare in the instrument's optical system, but post-mission measurements have revealed that at least a significant portion of the excessive signal was actually coming from contamination (e.g., dust) in the samples themselves. Since the samples are very well index matched (to ensure single scattering) and dust is not well index matched, the desired low angle signal from these samples is largely dominated by noise. Ironically, the instrument did indeed make low angle measurements, just not of the desired science. The instrument was able to make measurements down to $\theta=0.4^{\circ}$ (now felt to be ten times larger than the 


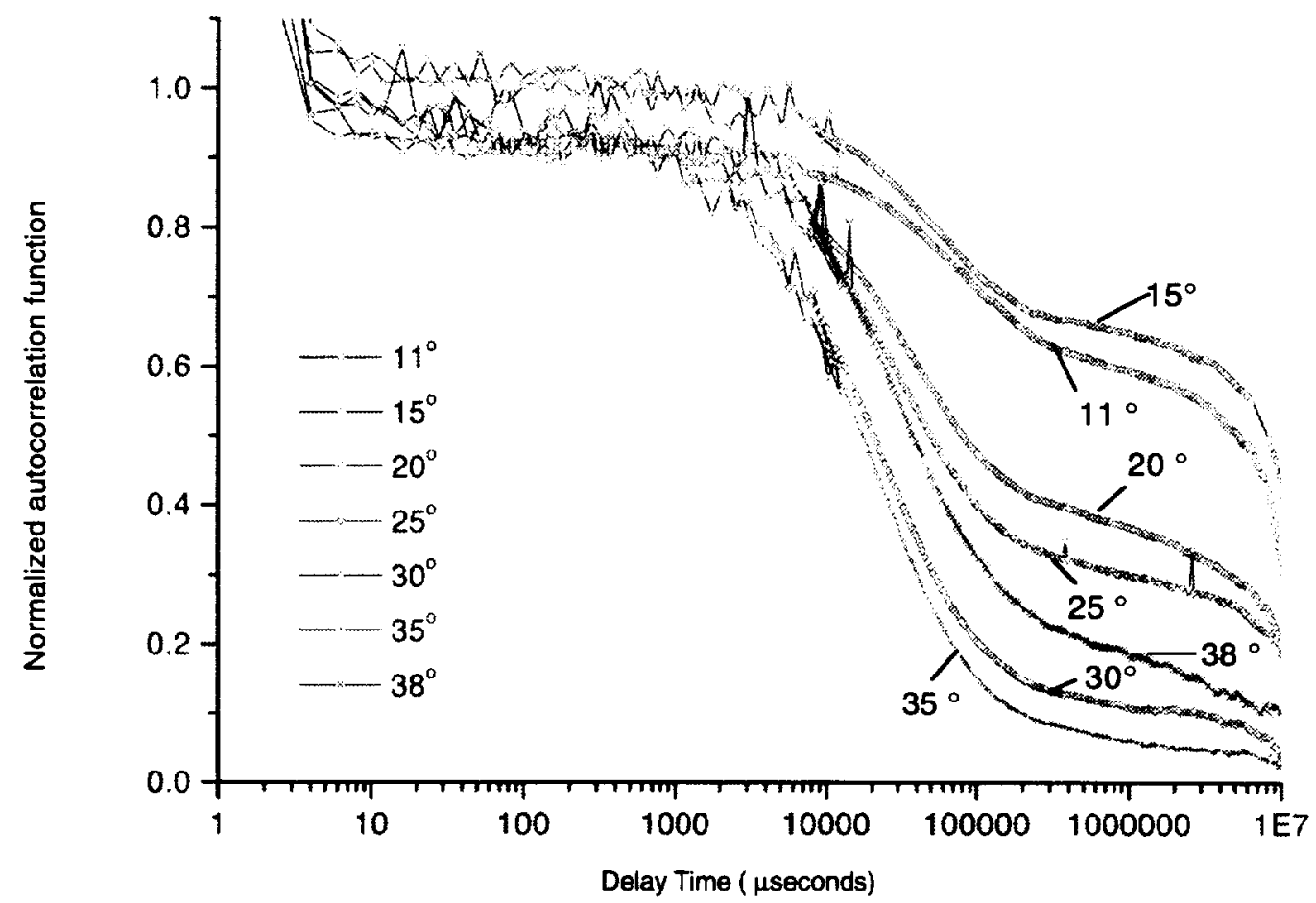

Fig. 8. Normalized autocorrelation functions for Sample 4 at seven scattering angles, from $11^{\circ}$ to $38^{\circ}$.

necessary minimum observable angle). Future low angle characterization of PMMA-based hard sphere colloids to determine crystallite nucleation and growth rates to required accuracies will require not only an instrument able to make a measurement down to at least $\theta=0.03^{\circ}$, but also samples purer than what were used in PHaSE.

Bragg images taken after crystallization is largely complete (e.g., 53,135 seconds (or $\approx 14.5$ hours) after shear melting of Sample 4) also provide measurement of the crystal lattice and crystal arrangement. A peak of $\theta=38.1^{\circ}$, as measured by 2-D Bragg scattering after 650,106 seconds (or $\approx 7.5$ days) for Sample 4 , reveals a lattice spacing of $863 \pm 4$ nanometers. The through-system angular resolution requirements to support the required accuracies for lattice constant, $\Delta \theta \leq 0.2^{\circ}$, were met as stated earlier for nucleation and growth. The shape of the Bragg peak (and its shoulders) for Sample 4 indicates Random Hexagonal Close Packed structure, with a slight amount of Face Centered Cubic, as evidenced by the "bump" that is forming over time in the trace at approximately $\theta=43^{\circ}$. SLS on this sample from $\theta=10^{\circ}$ to $150^{\circ}$ (Figure 7) yields good agreement with the 2-D Bragg data for peak locations at $\theta \leq 60^{\circ}$, and also provides valuable data on Bragg peaks beyond $60^{\circ}$.

DLS measurements at numerous scattering angles help characterize the Brownian fluctuations for each phase. As one complete rotation of the PHaSE sample cell during the measurements generates an ensemble of nearly 6000 samplings, the required accuracies for the diffusion factor and root mean square displacement were met. DLS was conducted for Samples 3, 4, and 5 at up to eleven different scattering angles from $11^{\circ}$ to $55^{\circ}$. Although the data at the four largest angles, $40^{\circ}, 45^{\circ}, 50^{\circ}$, and $55^{\circ}$, are noisy (probably due to the low optical power in the D\&S beam), the ensemble averaged autocorrelation functions at the lower angles exhibit the expected form. Typically, these ensemble averages contain either 500 or 1500 (approximately) independent 10-second correlations collected at the same number of 


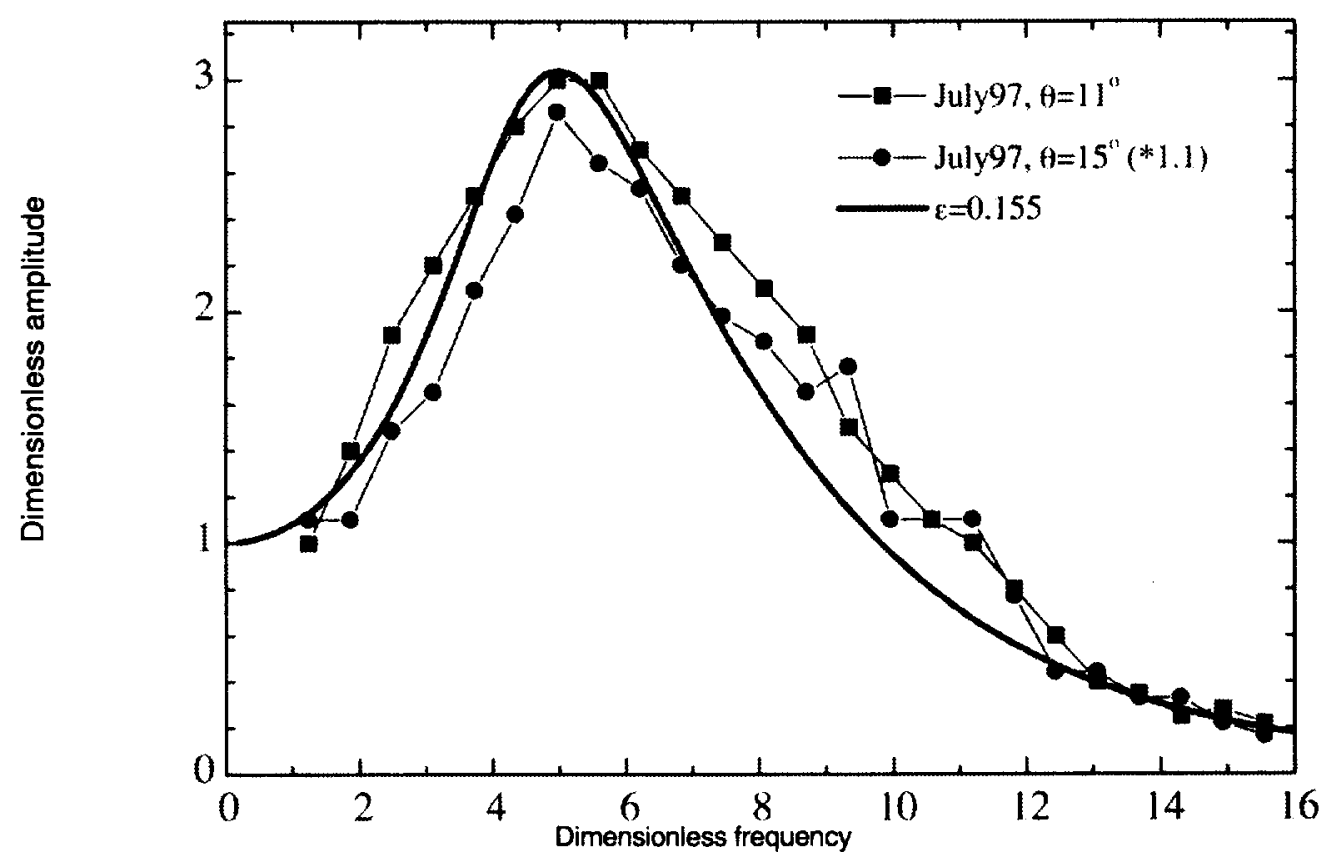

Fig. 9. Rheology investigation on Sample 3: normalized amplitude versus normalized frequency

discrete cell positions, achieved through rotating the sample cell by an $0.1^{\circ}$ increment between each measurement. (The number of discrete cell positions at which the measurement was made for each scattering angle, either 500 or 1500 , was determined by the PIs, based upon their priorities for the use of the on-orbit time.) This technique provides statistically independent sampling of the static speckle field on this non-ergodic system. Figure $\mathbf{8}$ shows autocorrelations of a crystalline sample (Sample 4) at seven scattering angles, from $11^{\circ}$ to $38^{\circ}$. The quality of the data in the range of $\tau \approx 10^{4}$ to $10^{5} \mu \mathrm{s}$ should yield good measurement of the diffusion rate. The scatter in the data at delays less than $5000 \mu \mathrm{s}$ is most likely due to the low optical power, but isn't expected to seriously compromise the measurement. A second DLS measurement technique utilized during flight, which measured the value of the asymptote at delay times up to ten minutes $\left(6 \times 10^{8} \mu \mathrm{s}\right)$, is expected to yield the root mean square displacement of the hard spheres.
DLS measurements taken at $15^{\circ}$ and $30^{\circ}$ while slowly oscillating the sample cell over a range of frequencies, provide very good quantification of the static elastic modulus and dynamic viscosity of the crystalline phase. The frequency sweep reveals the excitation of a resonance in the crystal, and has proven to be an excellent new technique for measuring the rheological properties of a colloidal crystal. As the sample cell drive frequency had a resolution of 0.1 Hertz, the required accuracies for the elastic constant and dynamic viscosity were met. Figure 9 is a plot of the dimensionless amplitude versus dimensionless frequency for Sample 3 showing that the empirical data from STS-94 appear to agree with theory, the $\varepsilon=0.155$ curve. As stated earlier, the location and width of the fundamental peak will provide a quantitative measurement of the shear modulus and dynamic viscosity of this hard sphere colloid.

In addition to the above tests, the operational flexibility of the instrument permitted the Pls to conduct two additional investigations on STS- 
94. These included utilizing DLS to study the nature of a nucleating crystal and performing shear alignment / shear yielding investigations on several samples. These investigations, conceived in near real time, enabled the experiment to exceed its pre-launch success metrics, and may yield valuable information with regard to the yield stress and shear melting of the crystalline solid. For the purposes of a space-based LSI, implementing these runs also served to validate the advantages both of a versatile flight instrument and of remote commanding. These real-time changes had no impact on flight crew resources, and allowed the science investigation plan to be optimized in response to immediate results. This clearly has demonstrated that such a method of operations will work, which is important for the extended, and somewhat "crew time" constrained, science operations that will characterize the International Space Station era.

\section{SUMMARY}

The Physics of HArd Spheres Experiment (PHaSE) utilized NASA Lewis Research Center's state-of-the-art light scattering instrument for microgravity experiments on complex fluids, and flew on board the Space Shuttle's Microgravity Science Laboratory (MSL-1) in 1997. Using colloidal systems of various concentrations of micron-sized plastic spheres in a refractive indexmatching fluid as test samples, light scattering experiments were conducted to measure the nucleation and growth rate of colloidal crystals as well as the structure, rheology, and dynamics of the equilibrium crystal. Together, these measurements support an enhanced understanding of the nature of the liquid-to-solid transition. The flight instrument provided the ability to make many types of measurements on each specimen. Although the breadth of the investigation was limited because half of the sample complement had bubbles, the flight experiment clearly demonstrated the value of microgravity in colloids research while providing detailed data on the balance of samples, the quality and quantity of which is expected to deepen the current understanding of the liquid to solid transition. The instrument performed 2D Bragg and low angle scattering from $\theta=0.4^{\circ}$ to $60^{\circ}$, dynamic and single-channel static scattering from $\theta=10^{\circ}$ to $170^{\circ}$, rheology using fiber optics, and white light imaging of the sample, all (except for the low angle measurement) to required accuracies. Excess signal on the screen at low angles contributed to the failure to accomplish the low angle science. Nevertheless, PHaSE provided both a rigorous test of the benefits of microgravity complex fluids research, and a timely microgravity demonstration of critical light scattering measurement techniques and hardware concepts - to set the stage for the next generation of light scattering flight instrumentation currently under design - while generating data already showing promise of interesting new scientific findings in the field of condensed matter physics. 


\section{REFERENCES}

1. J.P. Hansen and I. R. McDonald, Theory of Simple Liquids (Academic Press, London, 1986)

2. W. B. Russel, D. A. Saville, and W. R. Schowalter, Colloidal Dispersions (Cambridge U. Press, Cambridge, 1989).

3. P. M. Chaikin, and T. C. Lubensky, Principles of Condensed Matter Physics (Cambridge U.Press, Cambridge, 1995).

4. R. Car, "How hard spheres stack up," Nature (London) 385, 115-116 (1997).

5. C. Wu, "Gazing into crystal balls," Science News (Washington, D. C.) 151, 224-225, (1997).

6. J. Zhu, M. Li, R. B. Rogers, W. V. Meyer, R. H. Ottewill, STS-73 Space Shuttle Crew, W. B. Russel, and P. M. Chaikin, "Crystallization of hard sphere colloids in microgravity," Nature (London) 387, 883-885 (1997).

7. R. B. Rogers, W. V. Meyer, J. Zhu, P. M. Chaikin, W. B. Russel, M. Li, and W. B. Turner, "Compact laser light scattering instrument for microgravity research," Appl. Opt. 36, 74937500 (1997).

8. W. B. Russel, P. M. Chaikin, J. Zhu, W. V. Meyer, and R. B. Rogers, "Dendritic growth of hard sphere crystals," Langmuir 13, 3871-3881 (1997).

9. NASALaser Light Scattering Advanced Technology Development Workshop -1988, Proceedings of a workshop sponsored by the NASALewis Research Center, NASA CP-10033 (1989).
10. W. V. Meyer and R. R. Ansari, "A preview of a microgravity laser light scattering instrument," AIAA 91-0779 (1991).

11. C. T. Lant, A. E. Smart, D. S. Cannell, W. V. Meyer, and M.P. Doherty, "Physics of hard spheres experiment - a general purpose light scattering instrument, Appl. Opt. 36, 75017507 (1997).

12. The mention of brand names here and throughout the paper is for informational purposes only and does not imply that alternative commercial or non - commercial approaches do not exist.

13. R. G. W. Brown, J. G. Burnett, J. Mansbridge, and C. I. Moir, "Miniature laser light scattering instrumentation for particle size analysis," Appl. Opt. 29, 4159-4169 (1990).

14. P. N. Pusey, and R. J. A. Tough, "Particle interactions," in Dynamic Light Scattering: Applications of Photon Correlation Spectroscopy (ed. R. Pecora), (Plenum Press, New York, 1985), pp. 85-179.

15. J. M. Rallison, and E. J. Hinch, "The effect of particle interactions on dynamic light scattering from a dilute suspension," Fluid Mech 167, 131-168 (1986).

16. B. J. Berne, and R. Pecora, Dynamic Light Scattering, (Wiley-Interscience, New York, 1976).

17. B. Chu, Laser Light Scattering, (Academic Press, New York, 1991).

18. W. B. Russel, D. A. Saville, and W. R. Schowalter, p. 72.

19. W. B. Russel, D. A. Saville, and W. R. Schowalter, p. 432. 
The Physics of Hard Spheres Experiment on MSL-1: Required Measurements and Instrument Performance

\section{AUTHOR(S)}

WU-963-20-0B-00

Michael P. Doherty, Christian T. Lant, and Jerri S. Ling

\section{PERFORMING OAGANIZATION NAME(S) AND ADDRESS(ES)}

National Aeronautics and Space Administration

Lewis Research Center

Cleveland, Ohio 44135-3191
8. PERForming organization REPORT NUMBER

E-11133

\section{SPONSORINGMONITORING AGENCY NAME(S) AND ADDRESS(ES)}

National Aeronautics and Space Administration

Washington, DC 20546-0001
10. SPONSORINGMONITORING AGENCY REPORT NUMBER

NASA TM-1998-206978

AIAA-98-0462

\section{SUPPLEMENTARY NOTES}

Prepared for the 36th Aerospace Sciences Meeting sponsored by the American Institute of Aeronautics and Astronautics, Reno, Nevada, January 12-15, 1998. Michael P. Doherty and Jerri S. Ling, NASA Lewis Research Center; Christian T. Lant, ADF Corporation, 3003 Aerospace Parkway, Brook Park, Ohio 44142. Responsible person, Michael P. Doherty, organization code $6728,(216)$ 433-6641.

\section{DISTRIBUTIONAVAILABILTYY STATEMENT}

12b. DISTRIBUTION CODE

Unclassified - Unlimited

Subject Category: 29
Distribution: Nonstandard

This publication is available from the NASA Center for AeroSpace Information, (301) 621-0390.

13. ABSTRACT (Maximum 200 worda)

The Physics of HArd Spheres Experiment (PHaSE), one of NASA Lewis Research Center's first major light scattering experiments for microgravity research on complex fluids, flew on board the Space Shuttle's Microgravity Science Laboratory (MSL-1) in 1997. Using colloidal systems of various concentrations of micron-sized plastic spheres in a refractive index-matching fluid as test samples, illuminated by laser light during and after crystallization, investigations were conducted to measure the nucleation and growth rate of colloidal crystals as well as the structure, rheology, and dynamics of the equilibrium crystal. Together, these measurements support an enhanced understanding of the nature of the liquidto-solid transition. Achievement of the science objectives required an accurate experimental determination of eight fundamental properties for the hard sphere colloidal samples. The instrument design met almost all of the original measurement requirements, but with compromise on the number of samples on which data were taken. The instrument performs 2-D Bragg and low angle scattering from $0.4^{\circ}$ to $60^{\circ}$, dynamic and single-channel static scattering from $10^{\circ}$ to $170^{\circ}$, rheology using fiber optics, and white light imaging of the sample. As a result, PHaSE provided a timely microgravity demonstration of critical light scattering measurement techniques and hardware concepts, while generating data already showing promise of interesting new scientific findings in the field of condensed matter physics.

\section{SUBJECT TERMS}

Light scattering; Hard sphere; Bragg scattering; DLS; PCS; Crystal growth; Microgravity; Colloid

\begin{tabular}{|c|c|c|}
\hline $\begin{array}{c}\text { 17. SECURTY CLASSIFICATION } \\
\text { OF REPORT } \\
\text { Unclassified }\end{array}$ & $\begin{array}{c}\text { 18. SECURITY CLASSIFICATION } \\
\text { OF THIS PAGE } \\
\text { Unclassified }\end{array}$ & $\begin{array}{c}\text { 19. SECURITY CLASSIFICATION } \\
\text { OF ABSTRACT } \\
\text { Unclassified }\end{array}$ \\
\hline
\end{tabular}

NSN 7540-01-280-5500 